Voix et Images

volxetimages

\title{
Les livres parlent : de l'imposture et des imposteurs
}

\section{Pierre Milot}

Volume 11, numéro 1, automne 1985

\section{Naïm Kattan}

URI : https://id.erudit.org/iderudit/200542ar

DOI : https://doi.org/10.7202/200542ar

Aller au sommaire du numéro

Éditeur(s)

Université du Québec à Montréal

ISSN

0318-9201 (imprimé)

1705-933X (numérique)

Découvrir la revue

Citer cet article

Milot, P. (1985). Les livres parlent : de l'imposture et des imposteurs. Voix et Images, 11(1), 108-113. https://doi.org/10.7202/200542ar d'utilisation que vous pouvez consulter en ligne.

https://apropos.erudit.org/fr/usagers/politique-dutilisation/ 


\title{
Essai
}

\section{Les livres parlent: de l'imposture et des imposteurs}

\author{
par Pierre Milot, Université du Québec à Montréal
}

Si les livres parlent ${ }^{1}$, c'est que quelqu'un les fait parler et qu'il existe des conditions de possibilité pour que ce qu'ils disent soit entendu. Aussi, les trois livres dont il sera ici question accumulent les indices tendanciels de ce qu'est la situation actuelle de l'avant-garde littéraire, au Québec, à savoir l'état des agents qui y développent leurs stratégies discursives en s'autorisant d'un dispositif critique pour lequel ils anticipent de s'inscrire dans les processus de légitimation de l'institution littéraire.

Est-ce un hasard si deux de ces livres posent nominalement la question de l'imposture ${ }^{2}$ comme problématique de leur entreprise? Même si l'un (André Beaudet) la célèbre et l'autre (Michel Muir) la dénonce, l'un et l'autre s'en servent comme d'une audace intellectuelle et d'une provocation littéraire qui les placeraient du coup à l'écart, tant de l'avant-garde elle-même que du champ de la littérature instituée. Quant à Claude Beausoleil, plus enclin au consensus institutionnel, c'est au nom de la passion qu'il entend intervenir dans le discours critique sur la nouvelle poésie québécoise. Donc, quelque soit la posture adoptée (mais aussi à partir de cette position stratégique), ce qui nous intéresse dans chacun de ces ouvrages c'est ce qu'ils nous révèlent des conditions et des déterminations qui génèrent les relations entre l'institution littéraire et l'avant-garde instituante.

\section{Généalogie des objets consacrés et promotion des nouveautés}

Poète, critique, conférencier, professeur au collège Édouard-Monpetit, depuis 1978 Claude Beausoleil est chroniqueur de poésie au journal Le Devoir, peut-on lire sur la couverture arrière du recueil d'articles rassemblés sous le titre Les livres parlent, d'ailleurs préfacé par Jean Royer qui en tant que responsable des pages littéraires du Devoir en avait fait la commande à Beausoleil.

Dans un style cursif, Royer prévient le lecteur qu'ici le critique ne fait pas un bilan à froid mais une lecture à chaud... dans l'accueil des textes. Royer précise qu'il ne s'agit pas là de l'essai définitif sur la modernité des années 1970-1980, car soutient-il: il (Beausoleil) assume le destin fragile du travail journalistique et ses fragments critiques tirés de l'éphémère page de journal ont le grand mérite de faire confiance à la durée des textes des poètes. D'autant plus que Royer avoue s'ennuyer à la lecture des exégèses littéraires des savants du texte. On peut donc en déduire que le recueil d'articles (couvrant soixante et un auteurs québécois) de Beausoleil convient à la mesure de ses goûts. On se rappellera que Jean Royer avait aussi ouvert les pages littéraires du Devoir à l'avant-garde littéraire lors d'une série d'articles publiés sous le titre génétique "Les nouveaux écrivains et la critique» en 1979, de même qu'en 1980 suite au Colloque de La Nouvelle Barre du Jour qui avait consacré la dénomination nouvelle écriture. Ceci pour souligner que l'euphémisme concernant l'éphémère page de journal doit être retraduit 
dans l'ensemble des opérations de légitimation auxquelles se livrent les agents du champ littéraire (écrivains, critiques, spécialistes, journalistes).

Dans l'introduction de son recueil (qui constitue en fait le texte le plus long de l'ouvrage) Beausoleil écrit d'entrée de jeu: Mon histoire c'est l'histoire d'une passion. Une passion de lecture, une passion d'écriture. Une histoire étalée dans le secret des livres. Quelque chose comme une confidence, une affaire de coeur qui a pris le chemin de l'imprimerie (p. 13). En somme, Beausoleil est rétif à la critique littéraire dogmatique ou même de synthèse. Plus encore: il faut se laisser guider par les livres et leurs différentes versions du réel... la création est un chant intime qui parle à ceux qui l'écoutent.

Le paradoxe de cette procédure méthodologique, sorte d'hédonisme et de refus de la science évacuant les conditions structurales de production de l'écriture, c'est qu'elle repose sur un socle impressionniste contre lequel l'avant-garde formaliste des années 1970 (dont Beausoleil est l'un des agents) s'était méthodiquement opposée et contre lequel s'était érigée la sémiologie littéraire en vogue aux Herbes rouges et à La Barre du jour, au début des années 1970. C'est que les conditions sociales de production étant ce qu'elles sont, et le champ littéraire étant un marché où l'avant-garde se doit d'opérer un travail d'institutionnalisation au risque d'une dévaluation des profits symboliques accumulés durant sa phase inaugurale (phase d'agressivité sémantique et d'écarts stylistiques), Beausoleil n'a pas d'autre choix que d'opérer un certain effet de normalisation de la nouvelle poésie québécoise afin de lui assurer sa place dans la généalogie des objets consacrés et de ressouder l'intérêt générique de l'institution littéraire. Toutes les formes rhétoriques de son introduction et des articles de son recueil sont marquées par cette volonté de se conformer à la logique instituée: C'est en trente ans que toute la surface du paysage a basculé et que notre littérature et notre société sont passées d'un retard historique à une attitude de recherche et de changement, écrit-il pour légitimer la filiation généalogique de la modernité; du Refus Global, en passant par l'Hexagone, jusqu'aux années 1970 et 1980 (La Barre du Jour et Les Herbes rouges), ayant pris soin auparavant de remonter la filière jusqu'à Nelligan notre premier classique (p. 16-17). C'est ainsi que son discours critique sera traversé par une terminologie floue, sans délimitation rigoureuse sinon l'assignation récurrente à la nouveauté(qui, soit dit en passant, est la catégorie la plus divulguée sur le marché de la valeur littéraire).

Lorsqu'il parle des années 1980, Claude Beausoleil utilise la notion de nouveau lyrisme pour rendre compte des modalités énonciatives des textes produits par ceux et celles qui pendant les années 1970 avaient le plus articulé les positions de transgression (p. 27). Et c'est probablement là que le niveau d'analyse est le plus précaire, que les problèmes méthodologiques se posent, et que l'entreprise esquive sa fonction critique. Car comment expliquer que pour l'avant-garde formaliste des années 1970 le lyrisme constituait le style expressif de l'arrière-garde poétique et que dans les années 1980 le vécu, la mélancolie et l'intimité sont devenus les nouvelles unités de mesure de la modernité. De la modernité ou de la post-modernité? Encore ici l'usage de 
ce concept procède d'une formalisation confuse: l'importation du concept de post-modernité (en provenance de la critique universitaire américaine et retraduit en France par Lyotard) appliqué à la nouvelle écriture québécoise dénote chez Beausoleil une méconnaissance discursive des enjeux de ce débat (ce qu'il est convenu d'appeler le débat Lyotard/Habermas sur les rapports entre modernité et post-modernité). À trop vouloir aller de changement en changement (p. 29), on risque de s'embrouiller dans les toutes dernières définitions de la scientificité parisienne et par conséquent d'être disqualifié sur le marché des échanges poétiques. Heureusement que l'institution littéraire est le repos du guerrier.

Si Claude Beausoleil apporte une contribution au consensus institutionnel, par cette série d'articles couvrant soixante et une figures littéraires (de Rina Lasnier à Nicole Brossard, de Gaston Miron à François Charron, de Paul-Marie Lapointe à Roger Des Roches), il considère toutefois que certains spécialistes, dont la tâche est de codifier les valeurs génériques des produits légitimés par l'institution, commettent des erreurs de jugement en ce qui concerne la nouvelle poésie des années 1970 et 1980. C'est ainsi qu'il reproche aux auteurs de l'anthologie La Poésie québécoise des origines à nos jours (Laurent Mailhot et Pierre Nepveu) d'avoir mal officialisé le statut de certaines modalités énonciatives incorporées à ce qui (selon lui) constitue la valeur distinctive de la nouvelle écriture (la modernité). Mais Beausoleil reconnaît sans réluctance l'utilité de ce genre d'instruments d'inculcation pédagogique que sont les anthologies et les dictionnaires: en dépit, semble$\mathrm{t}$-il, des fonctions de contrôle et de correction des écarts et des transgressions qui sont assignées à ces entreprises de normalisation: il est même extrêmement nécessaire que toute littérature nationale autonome s'affirme à travers ces travaux de morceaux choisis, anthologies, monographies, thèses universitaires, etc. (p. 230). Ceci donne la mesure de la complexité des médiations qui structurent le champ littéraire et des processus de légitimation qui activent l'espace institutionnel.

\section{Une stratégie de distinction: la posture de l'exclu}

André Beaudet occupe une position de retrait et écrit à partir d'un lieu tordu. C'est du moins ce qu'il affirme dans le recueil d'essais littéraires qu'il vient de faire paraître sous le titre Littérature, L'imposture. Sur la couverture arrière du livre on apprend qu'il y sera question des malentendus qu'il entretient avec l'institution littéraire et la nouvelle écriture, de même que de la position intenable qui le rend intraitable. Et comme pour concrétiser, par un effet de réalisme, le caractère abyssal de cette pratique d'écriture qu'est la sienne, Beaudet a placé une dédicace à sa famille au nom de l'étrange aventure nocturne dont elle est à la fois partie prenante et exclue. Un long épigraphe de Nietzche sur la parole du solitaire précède le texte de présentation de l'ouvrage.

Tout compte fait, Beaudet se veut inquiétant et utilise tous les procédés rhétoriques de l'étrangeté du monde, du moins le monde que lui révèle son expérience subjective de la littérature. D'autorité le lecteur est prévenu de la rareté des objets discursifs qu'il a devant les yeux et de l'exclusion institutionnelle de l'auteur qui se veut le bouc émissaire des jeux de relations et des 
enjeux performatifs du champ littéraire. Ce qui, dans le système de classement des thèmes et des styles littéraires, peut être désigné comme la posture de l'exclu: posture symbolique qui est aussi chevillée à une stratégie de distinction sur le marché concurrentiel de la littérature.

Beaudet présente ses textes comme une riposte contre la fatigue culturelle et l'amnésie actuelle d'une certaine modernité qui s'empoisse jusqu'à l'aphasie du désir... Si je m'accommode tant bien que mal de la "nouvelle écriture » à laquelle on m'a collé, fiché, assigné... je dois rire de tout effet de surveillance et de vigilance édicté depuis peu à mon endroit (p. 12). Face à cette situation d'écrivain maudit, Beaudet a décidé de se jouer des mille et une impostures qui le guettent sur son chemin (l'imposture étant généralisée), et de se laisser aller à l'errance. D'où la volonté irrémédiable d'affirmer son droit à la dissonance dans des textes en rupture de contrat. Ce qu'il condense dans une formule toute lacanienne: je ne suis jamais là où l'on me cherche, on ne me trouve pas où je suis, donc je suis toujours là comme si je n'y étais pas (p. 13).

Chose certaine, si André Beaudet écrit dans l'autre monde, ses textes sont édités (et même lus publiquement) dans le monde social. Chacun des essais de cet ouvrage (à l'exception des "Fragments d'imposture») a fait l'objet soit d'une publication dans une revue instituée (réponses à un questionnaire de Liberté), soit d'une communication à un Salon du livre, à un Colloque universitaire, à un Musée ou sur les ondes de Radio-Canada. Le moins que l'on puisse dire c'est que si André Beaudet n'est jamais là où on le cherche et que l'on ne le trouve pas où il est, il semble bien qu'il sache où aller et quand il le faut. En définitive, l'expérience de la littérature l'a doté d'un certain sens du placement. Ce qui pourrait tenir dans la formule suivante: Beaudet n'est pas de cette nouvelle écriture où on le classe communément, mais c'est quand il n'y est pas qu'il en est un des plus éminents représentants.

Dans le texte consacré à Nelligan (titré «Nelligan's Fake», à partir d'un jeu de langage opéré avec le Finnegans Wake de Joyce), Beaudet se livre à la reconstitution du mode d'exclusion dont Nelligan fut l'objet dans le discours normatif de Lionel Groulx: Nelligan, parasite, est expulsé du discours de Groulx (p. 37). À la mission de socialisation des âmes de l'un, Beaudet oppose l'errance intime et désespérée de l'autre. Ce qui l'autorise à faire observer au lecteur que Lionel Groulx vient occuper le devant de la scène québécoise pendant les quarante années de réclusion de Nelligan (p. 37). C'est ce type de régularité discursive opposant l'espace social à l'espace poétique dans un dualisme métaphysique irréconciliable qui structure le discours critique de Beaudet: comme si ces deux espaces n'étaient pas le produit historique de systèmes de convictions et de pratiques de croyances surdéterminées par le projet de la modernité occidentale. D'ailleurs Beaudet trahit quelque peu son propos lorsqu'il trace la filiation de ceux qui subiront, à la suite de Nelligan, l'expérience désespérée de la poésie: Alain Grandbois, Saint-Denys Garneau, Claude Gauvreau, François Charron. Chacun de ces poètes a opéré (opère encore) l'expansion générique du champ littéraire au Québec. Expansion qui s'est manifestée par la mise en place de mécanismes institutionnels constitutifs de l'espace social. 
Donc, l'ensemble des essais de cet ouvrage sont utilisés par André Beaudet pour construire l'image qu'il voudrait qu'on se fasse de lui comme écrivain: et pour ce faire, il nous indique le repérage rhétorique de son adhésion à la valeur littéraire, soit sa vénération à l'égard d'auteurs auxquels il s'identifie (Lacan, Joyce et Kafka). Il est tout de même curieux qu'à partir d'une telle position, Beaudet puisse se lancer dans une polémique avec les produits concurrents de l'avant-garde québécoise, dans les termes suivants: Dans ce qui se prend actuellement au Québec pour le texte d'avant-garde, nous assistons à un retour de la citation d'autorité et sa répétition grégaire... Vision formaliste qui réduit son champ d'exploration à sa plus simple notation (le plus souvent anecdotique) (p. 48). Il y aurait donc des références légitimes (les siennes) et des citations dévaluées (celles des autres).

\section{Le pamphlétaire sacerdotal et le marché littéraire}

Le pamphlet publié par Michel Muir, et édité par Louise Courteau, sous le titre provocateur Poètes ou imposteurs, relève du ressentiment érigé en méthode. D'où le risque d'obscurantisme généré par ce genre d'entreprise quand, en plus, la compétence technique fait défaut. Et si la maison d'édition qui a lancé un tel ouvrage sur le marché se propose de s'impliquer dans une stratégie de promotion du même niveau, cela soulève la question de la valeur littéraire et de ses objets.

Michel Muir a décidé de faire cuvre de salubrité intellectuelle par la dénonciation de l'imposture de l'écriture poétique, soit la production des Herbes rouges (de France Théoret à Claude Beausoleil en passant par François Charron). Ce genre de pamphlet aurait pu être d'un intérêt spécifique, compte tenu de la position symbolique occupée par Les Herbes rouges dans le champ littéraire et plus particulièrement dans la sphère de l'avantgarde instituante, d'autant plus qu'avec les conflits de légitimité qui s'y jouent depuis la polémique entre modernité et religiosité il y aurait eu place pour un travail d'objectivation de ce type de productions et des stratégies de distinction auxquelles se livrent les poètes de la nouvelle écriture. C'est que Muir a ses propres ambitions et que ses prétentions à la poésie sont de l'ordre des lois éthiques, organiques et souverainement rationnelles qui gouvernent le cosmos (p. 22). De sorte que tout le mode énonciatif de son discours critique est structuré autour d'un effet sacerdotal: La désobéissance aux lois éternelles de la pensée provoque une discontinuité radicale manifeste entre la conscience et le monde... Je me contenterai de rappeler que l'Homme ne recouvre sa liberté que dans la soumission aux règles cosmogoniques... et ne peut poursuivre l'itinéraire intracirconlocutoire de la pensée qu'une fois qu'il accepte d'épouser son ascension (p. 22-23).

C'est au nom d'une sémiologie spirituelle que Muir entend mener son rituel de stigmatisation contre la poésie du bidet et des draps souillés, la mise en scène des inclinaisons homosexuelles d'André Roy, la décadence actuelle et le relâchement intellectuel, en somme les parties génitales de l'écriture poétique... des impuissants de la plume. Ce pourrait être les nouveaux Chants de Maldoror (moins la compétence technique) si Muir ne s'en prenait pas avec une telle fougue au culte du satanisme et aux cérémonies copulatoires transposées dans le domaine de l'écrit. 
Le livre de Muir n'ayant pas reçu l'attention escomptée et les profits anticipés par la recension critique, l'éditrice Louise Courteau a décidé d'agir: un placard publicitaire sous forme de lettre adressée à Jean Royer critique littéraire au Devoir, et paru effectivement dans le journal en question (22 juin 85, p. 35). Louise Courteau veut dénoncer la censure opérée par le critique littéraire du Devoir dont les affinités avec l'idéologie poétique dominante expliqueraient son absence de vue sur la poésie authentique. Michel Muir et sa maison d'édition fourbissent leurs armes dans le jeu de positions du marché littéraire: cela est légitime puisqu'il y existe une libre circulation des produits poétiques. Quant à leur insertion dans les processus de légitimation, tant de la littérature institutée que de l'avant-garde instituante, c'est autre chose. Michel Muir sera peut-être le véritable outsider de la poésie québécoise.

1. Claude Beausoleil, Les livres parlent, les Écrits des Forges, Trois-Rivières, 1984.

2. André Beaudet, Littérature, L'imposture, Les Herbes rouges, Montréal, 1984. Michel Muir, Poètes ou imposteurs?, Louise Courteau Éditrice, Montréal, 1985. 